\title{
OPEN Distinct trans-placental effects of maternal immune activation by TLR3 and TLR7 agonists: implications for schizophrenia risk
}

\author{
Jaedeok Kwon ${ }^{1,2}$, Maria Suessmilch², Alison $\mathrm{McColl}^{2}$, Jonathan Cavanagh ${ }^{2,3}$ \& \\ Brian J. Morris ${ }^{1,3}$
}

Exposure to infection in utero predisposes towards psychiatric diseases such as autism, depression and schizophrenia in later life. The mechanisms involved are typically studied by administering mimetics of double-stranded (ds) virus or bacterial infection to pregnant rats or mice. The effect of single-stranded (ss) virus mimetics has been largely ignored, despite evidence linking prenatal ss virus exposure with psychiatric disease. Understanding the effects of gestational ss virus exposure has become even more important with recent events. In this study, in pregnant mice, we compare directly the effects, on the maternal blood, placenta and the embryonic brain, of maternal administration of ds-virus mimetic poly I:C (to activate Toll-like receptor 3, TLR3) and ss-virus mimetic resiquimod (to activate TLR7/8). We find that, $4 \mathrm{~h}$ after the administration, both poly $\mathrm{I}: \mathrm{C}$ and resiquimod elevated the levels of IL- $6, \mathrm{TNF} \alpha$, and chemokines including CCL2 and CCL5, in maternal plasma. Both agents also increased placental mRNA levels of IL- 6 and IL-10, but only resiquimod increased placental TNF $\alpha$ mRNA. In foetal brain, poly I:C produced no detectable immune-response-related increases, whereas pronounced increases in cytokine (e.g. Il-6, Tnf $\alpha$ ) and chemokine (e.g. $\mathrm{Ccl2}, \mathrm{Ccl5})$ expression were observed with maternal resiquimod administration. The data show substantial differences between the effect of maternal exposure to a TLR7/8 activator as compared to a TLR3 activator. There are significant implications for future modelling of diseases where maternal ss virus exposure contributes to environmental disease risk in offspring.

Maternal bacterial and viral infections during pregnancy increase the risk of psychiatric disease in the offspring exposed to the infection in utero. This is the case for autism spectrum disorders, major depressive disorder ${ }^{1,2}$ and schizophrenia ${ }^{3-5}$. The mechanisms involved remain unclear, but are likely to involve the exposure of the developing foetal brain to either the infectious agent itself, or to cytokines and chemokines released from maternal or placental tissues as part of the innate immune response. Direct exposure of the developing foetal CNS to the infectious agent is possible, as the agents most strongly linked by epidemiological evidence to schizophrenia risk (rubella virus, influenza virus and the parasite Toxoplasma gondii) ${ }^{3-6}$, are all able to penetrate the placenta to invade the foetal environment ${ }^{7-9}$. Whatever the mechanism, the infectious agent or immune response molecules impact upon differentiating or migrating neurons e.g. inter-neurons, and can affect microglia in the developing CNS with consequences for processes such as synaptic pruning ${ }^{10}$. Exactly how these processes operate to raise risk of psychiatric disease remains uncertain.

Maternal immune activation models typically involve pregnant mice being injected with the bacterial mimetic lipopolysaccharide (LPS), or the double-stranded (ds) virus mimetic polyinosinic: polycytidylic acid (poly I:C). The consequences for the neurochemical and behavioural phenotype of the offspring can then be evaluated. A variety of disease-relevant changes in gene expression and social, affective and cognitive behaviours have been detected in offspring at various ages ${ }^{11-13}$, although there have also been concerns about the reproducibility of some of the data with poly I:C, due to a variety of technical factors (e.g. batch variation, endotoxin contamination $)^{14,15}$.

${ }^{1}$ Institute of Neuroscience and Psychology, College of Medical, Veterinary and Life Sciences, University of Glasgow, Glasgow G12 80Q, UK. Institute of Inflammation and Immunity, University of Glasgow, Glasgow, UK. ${ }^{3}$ These authors contributed equally: Jonathan Cavanagh and Brian J. Morris. ${ }^{\circledR}$ email: Brian.Morris@glasgow.ac.uk 
LPS reproduces the effects of bacterial infection by stimulating the Toll-like receptor (TLR) TLR4, while poly I:C reproduces the effects of ds-virus infection by stimulating TLR3. Ss-viruses stimulate TLR7 or TLR8 to induce an immune response. However, despite the fact that some of the prenatal infectious agents most strongly linked to schizophrenia risk (rubella virus and influenza virus) are ss-viruses, models of maternal immune infection with ss-virus mimetics such as resiquimod or imiquimod, which stimulate TLR7/8 ${ }^{16,17}$ are rarer. Similarly, Toxoplasma gondii also induces an innate immune response via TLR $7^{18,19}$, while ds-viruses such as Herpes simplex II are not robustly linked to schizophrenia risk ${ }^{20}$. Furthermore, some recent evidence in mice suggests that the behavioural consequences for offspring exposed to TLR7 stimulation in utero may be different to the effects of maternal TLR3 or TLR4 stimulant exposure ${ }^{21}$.

Our research question in this study was: what are the differences in the cytokine/chemokine responses to the commonly-used ds viral mimic versus ss viral mimic. We have directly compared the most commonly used strategy for studying the effects of MIA (poly I:C administration in mice) with a novel strategy using resiquimod to mimic ss- virus infection.

\section{Methods}

Experimental design. Poly I:C is frequently used in mice to model MIA. Concerns have been raised recently about variability in its effects, due partly to possible endotoxin contamination and batch variation ${ }^{14,15}$. The dose used is most commonly $20 \mathrm{mg} / \mathrm{kg}$, and the gestational time point is generally E9, E12.5 or E17, with E12.5 the most frequent choice, as it is argued to correspond in various ways to mid-gestation time in humans $s^{1,11-13}$. We used poly I:C of the specification that reportedly gives the most robust results ${ }^{14,15}$, and selected resiquimod as the ss-virus mimetic comparator. While imiquimod has recently been used to study the consequences for CNS function of ss-virus infection ${ }^{22,23}$, and to assess the behavioural effects in offspring of MIA $^{21}$, apart from acting as a ss-virus mimetic, imiquimod also has direct actions on adenosine receptors that complicate interpretation of its effects ${ }^{24,25}$. In addition, imiquimod is selective for TLR7, whereas resiquimod activates both TLR7 and TLR $8^{26}$. Considering that the epidemiological evidence for psychiatric disease risk implicates maternal ss-virus infection, without discriminating between TLR7 and TLR8 mediation, resiquimod seems to be the better choice to maximise construct validity in future models of environmental contribution to psychiatric disease risk.

Resiquimod has been used systemically in mice on occasion, producing an immune response at doses between 1 and $10 \mathrm{mg} / \mathrm{kg}^{27-30}$. McAllister et al. ${ }^{31}$ describe equivalent (and substantial) serum TNFa responses to high doses of $30 \mathrm{mg} / \mathrm{kg}$ poly I:C and $10 \mathrm{mg} / \mathrm{kg}$ resiquimod in mice. We therefore selected $2 \mathrm{mg} / \mathrm{kg}$ as a moderate dose of resiquimod to compare with the standard dose of $20 \mathrm{mg} / \mathrm{kg}$ poly I:C. This study is reported in accordance with ARRIVE guidelines (https://arriveguidelines.org).

Maternal immune activation and sample collections. In vivo experiments were performed according to Home Office (UK) guidance and regulations, and all studies were approved by the University of Glasgow Animal Welfare Ethical Review Board. Wild type (WT) mice (C57/BL6, female) were purchased from Envigo. Mice were time mated separated following day, and if the pregnancy was confirmed the day was given as E0. Female mice were weighed and monitored for 12 days. All mice were aged 7-9 weeks and weighed $30 \pm 1 \mathrm{~g}$ at the point of experiment (12.5 days pregnant). In order to induce maternal immune activation (MIA), three treatments, vehicle (PBS, $2 \mathrm{ml} / \mathrm{kg}$, Gibco 14190144), poly I:C $(20 \mathrm{mg} / \mathrm{kg}$ of a $10 \mathrm{mg} / \mathrm{ml}$ solution in PBS, LMW, Invivogen tlrl-picw, LOT \#11C21-MM), resiquimod ( $2 \mathrm{mg} / \mathrm{kg}$ of a $1 \mathrm{mg} / \mathrm{ml}$ solution in PBS, Invivogen tlrl-r848, LOT\#18L21-MM), were administered subcutaneously on E12.5 between 9:00 and 11:00 a.m. and their conditions were monitored to ensure that there was no sign of any severe sickness symptoms or abnormalities.

Four hours following injections, the pregnant dam was culled with the $\mathrm{CO}_{2}$ euthanasia. The maternal blood was collected via right atrium into an EDTA-coated syringe. The blood was injected into an EDTA-coated 1.5 micro centrifuge tube containing $100 \mu \mathrm{l}$ EDTA and shaken. Experimental samples were collected. Following centrifugation at $10,000 \mathrm{~g}$ at $4{ }^{\circ} \mathrm{C}$ for $10 \mathrm{~min}$, the supernatant (plasma) was frozen at $-80{ }^{\circ} \mathrm{C}$ for further use. Once the blood collection was done, the right atrium was cut, and PBS perfusion was carried out. After the PBS perfusion, maternal placentae and embryos were dissected and collected. All tissues were pre-processed and stored until further use. More details of sample preparation procedures are described in separate sections. All experiments were reviewed by the University of Glasgow ethical committee and were performed under the authority of UK Home Office License.

RT-qPCR. Collected tissues after MIA experiment were originally stored in RNAlater at $-80{ }^{\circ} \mathrm{C}$. Before extracting total RNA from the tissues, they had to be completely thawed in RT and RNAlater had to be removed. Under RNase-fee condition, the collected tissues were homogenised using the TissueLyser LT (Qiagen) in $600 \mu \mathrm{l}$ of prepared RLT lysis buffer (Qiagen). Total RNA was extracted from the stabilised tissues using an RNeasy mini kit (Qiagen, 79254) with additional DNase I (Qiagen, 79254) as per manufacturer's instruction. The RNA concentration was determined by a Nanodrop DeNovix DS-11 + Spectrophotometer. RNA was reverse-transcribed to cDNA using High-capacity RNA-to-cDNA ${ }^{\text {tx }}$ kit (Applied Biosystems, 4387406) according to the manufacturer's instruction. cDNA concentration was normalised by using equal quantity of RNA (normalised to $1 \mu \mathrm{g} / \mathrm{ml}$ ).

Gene expression in the tissues was quantified Fast SybrGreen ${ }^{\mathrm{Tm}}$ master mix (Applied Biosystems, 4385612) for each target using the QuantaStudio7 (Thermo Fisher Scientist). Samples were run in triplicate on 384 well qPCR plates (Applied Biosystems, 4309849), using $1 \mu \mathrm{g}$ of cDNA, and the levels of the target genes were normalised to a geometric mean of two housekeeping genes $(G a p d h, T b p)$. Relative differences in target gene expression were determined using absolute quantification method. Primer sequences are provided in Supplementary Table 2. 
Multiplex/Luminex. The concentration of 9 cytokines and chemokines with well-characterised roles in innate immune responses were measured in maternal plasma by a Multiplex/Luminex (Merck, MCYTMAG-70 K-PX32) according to the manufacturer's instruction. Measurements were taken (LUMINEX 200 ${ }^{\circ}$ ), operated via Bio-Rad's Bio-Plex software version 6.1 ${ }^{\text {mi }}$ (https://www.bio-rad.com/en-uk/category/bio-plex-softw are? ID $=45938 \mathrm{~d} 9 \mathrm{~d}-\mathrm{c} 2 \mathrm{ec}-4 \mathrm{ae} 4-9 \mathrm{ed} 3-\mathrm{e} 7358 \mathrm{a} 98 \mathrm{~d} 30 \mathrm{~b})$. The beads were read determining the mean fluorescence intensity (MFI) $)^{32-34}$ and the data were accepted if the duplicated samples vary (CoV) were less than $15 \%$.

Statistical analysis. All statistical analyses were carried out using Minitab 19 Statistical Software and all data were reported as group mean \pm S.E.M. Luminex data were Box-Cox transformed prior to one-way analysis of variance (ANOVA) with post hoc Fisher LSD tests to correct for multiple comparisons. Non-normally distributed RT-qPCR data were log transformed, followed by two-way ANOVA with post Tukey tests. A $p$-value of $<0.05$ was considered significant.

Ethics approval and consent to participate. In vivo experiments were performed according to Home Office (UK) regulations, and were approved by the University of Glasgow Ethical Review Board.

Consent for publication. Not applicable.

\section{Results}

Protein level changes in maternal plasma. Following poly I:C or resiquimod administration there were increases in the levels of immune molecules in maternal plasma, compared to vehicle administration. Of the 9 cytokine/ chemokines tested, IL-6, TNF, IL-10, CCL2, CCL5, CXCL10, and LIF (leukaemia inhibitory factor) were all elevated following both poly I:C and resiquimod administration: (Fig. 1). Maternal plasma from mice injected with resiquimod, but not poly I:C, also showed significantly upregulated CCL11 and CXCL1, (Fig. 1). In general, the effects of poly I:C showed a greater variability in the magnitude of cytokine/chemokine induction, compared to resiquimod.

Transcription level changes in placentae. The data from maternal plasma clearly confirm that the experimental conditions caused an immune response in the dams. We next assessed the response of placental tissue to the immune stimuli, measuring mRNA levels so that changes detected can be unequivocally ascribed to the placenta, as opposed to maternal plasma.

Resiquimod administration elevated expression of most of inflammatory cytokine and chemokine mRNAs (Tnf- $\alpha, C c l 5, C c l 11$ and $C x c l 1$ ) without affecting Ccl2 and Cxcl12 mRNA levels (Fig. 2).

Poly I:C induced Il-6, Il-10, and Cxcl10 mRNA levels, but changes were not observed in any other genes (Fig. 2A,C,H).While CCL2 in maternal plasma was significantly induced by both poly I:C and resiquimod injection (Fig. 1D), placental Ccl2 mRNA was not changed by either MIA condition compared to vehicle (Fig. 2D). Overall, the results suggest that while the response to poly I:C and resiquimod in maternal plasma is rather similar, the response of placental tissue to poly I:C is much more limited compared to the response to resiquimod.

Transcription level changes in foetal brains. The same cytokines and chemokines mRNAs were measured in foetal brain tissue $4 \mathrm{~h}$ after MIA (Fig. 3). Foetal brains from MIA caused by resiquimod showed significantly increased levels of most of cytokine and chemokine mRNAs compared to vehicle administration. However, the foetal brain tissue from poly I:C MIA did not show any induction of immune molecule genes.

Immune cell markers. There is considerable evidence for microglial activation in foetal brain following MIA with poly I: $\mathrm{C}^{35-38}$. We tested possible altered expression of microglial markers in foetal brain tissue after MIA.

The results showed that the expression of Aif1 (Iba-1) was significantly increased by resiquimod, but not by poly I:C (Fig. 4C). In contrast, Tmem 119 and $C x 3 \mathrm{cr} 1 \mathrm{mRNA}$ levels were down regulated by resiquimod (Fig. 4A,B). Noticeably, poly I:C caused a significant reduction in the levels of Tmem 119 mRNA, but not $C \times 3 \mathrm{cr} 1$ mRNA. Ccr 2 mRNA was decreased by both stimuli for MIA, although resiquimod's impact was greater than poly I:C (Fig. 4D). Ly6c2 mRNA levels were not affected by MIA (Fig. 4E).

In addition, due to its frequent link to environmental risk for psychiatric disease, we also monitored the expression of $B d n f$. There were no changes in $B d n f$ expression in foetal brain, but resiquimod exposure decreased $B d n f$ mRNA levels in placenta (Supplementary Fig. 1).

Sex effects. Foetal development is affected by $\operatorname{sex}^{39,40}$. In order to identify any sex-dependent influence on the foetal immune response, the embryos' sex was determined by qPCR, using the sex-specific Xist gene ${ }^{41}$. High Xist expression indicated female and low expression male (Supplementary Fig. 2), and suggested that the samples were well-balanced for sex within a treatment group. No statistically significant interaction of sex with the effect of MIA was observed for any of the markers.

\section{Discussion}

This study provides a direct comparison of the pattern of immune response to poly I:C and resiquimod in maternal, placental and foetal brain compartments. It is clear that their patterns of immune activation are different, and the differences are important for future studies of MIA effects on offspring. 

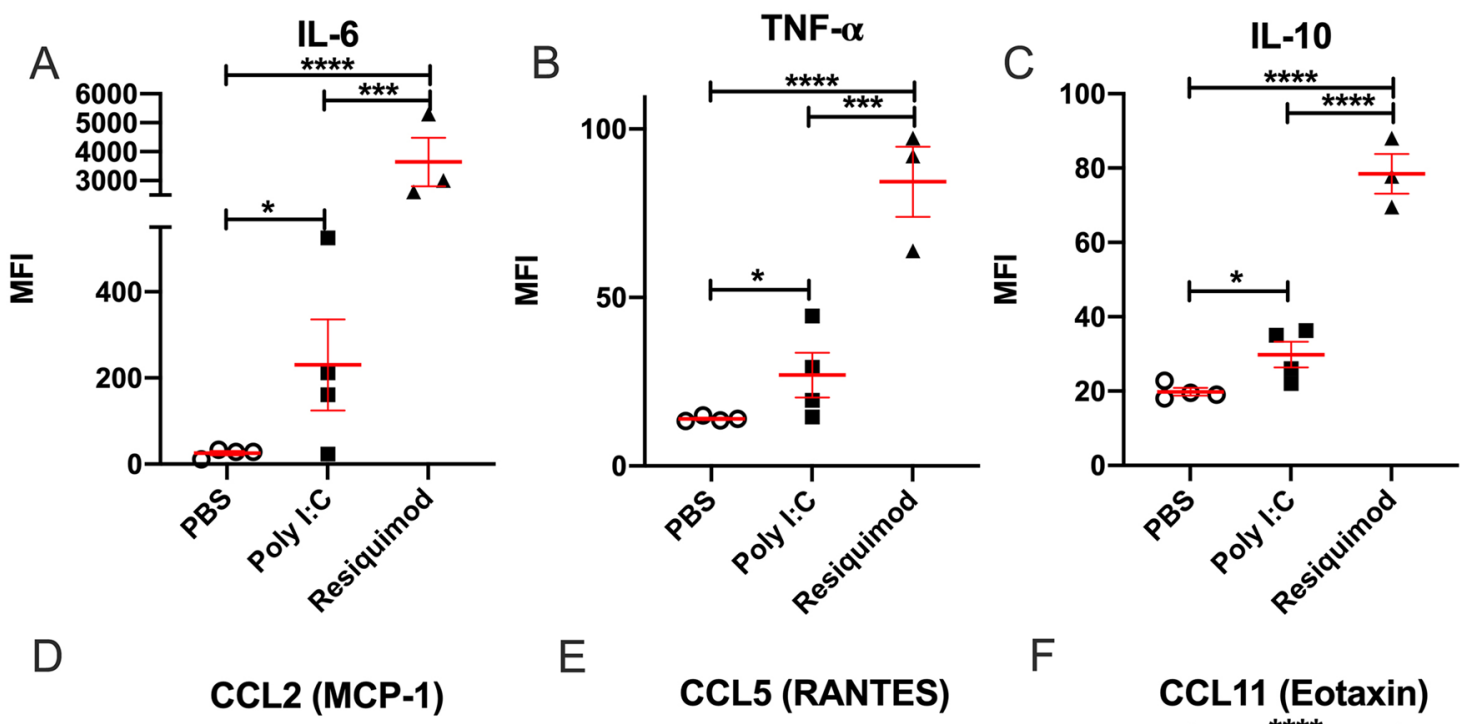

\section{E}
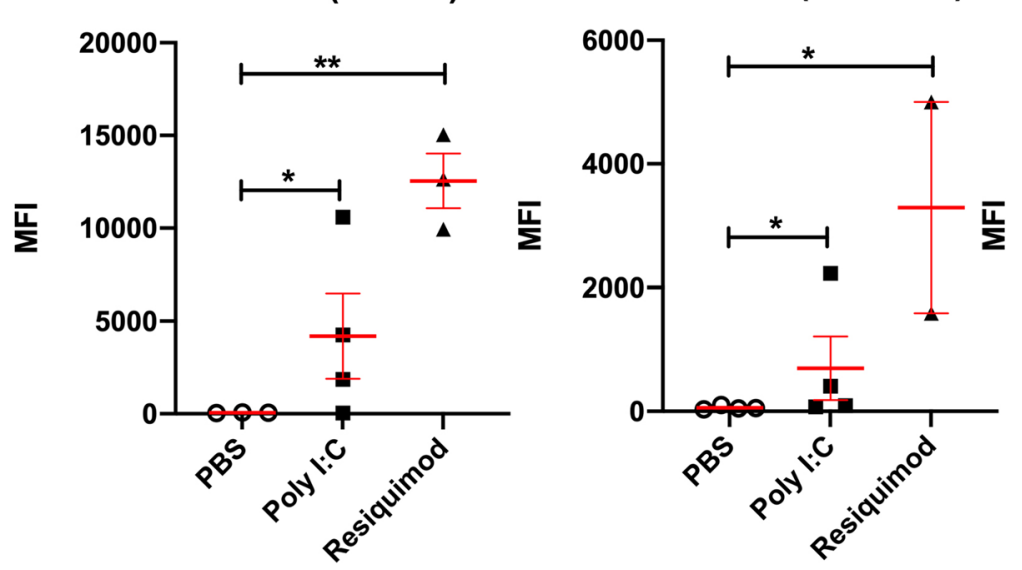

F
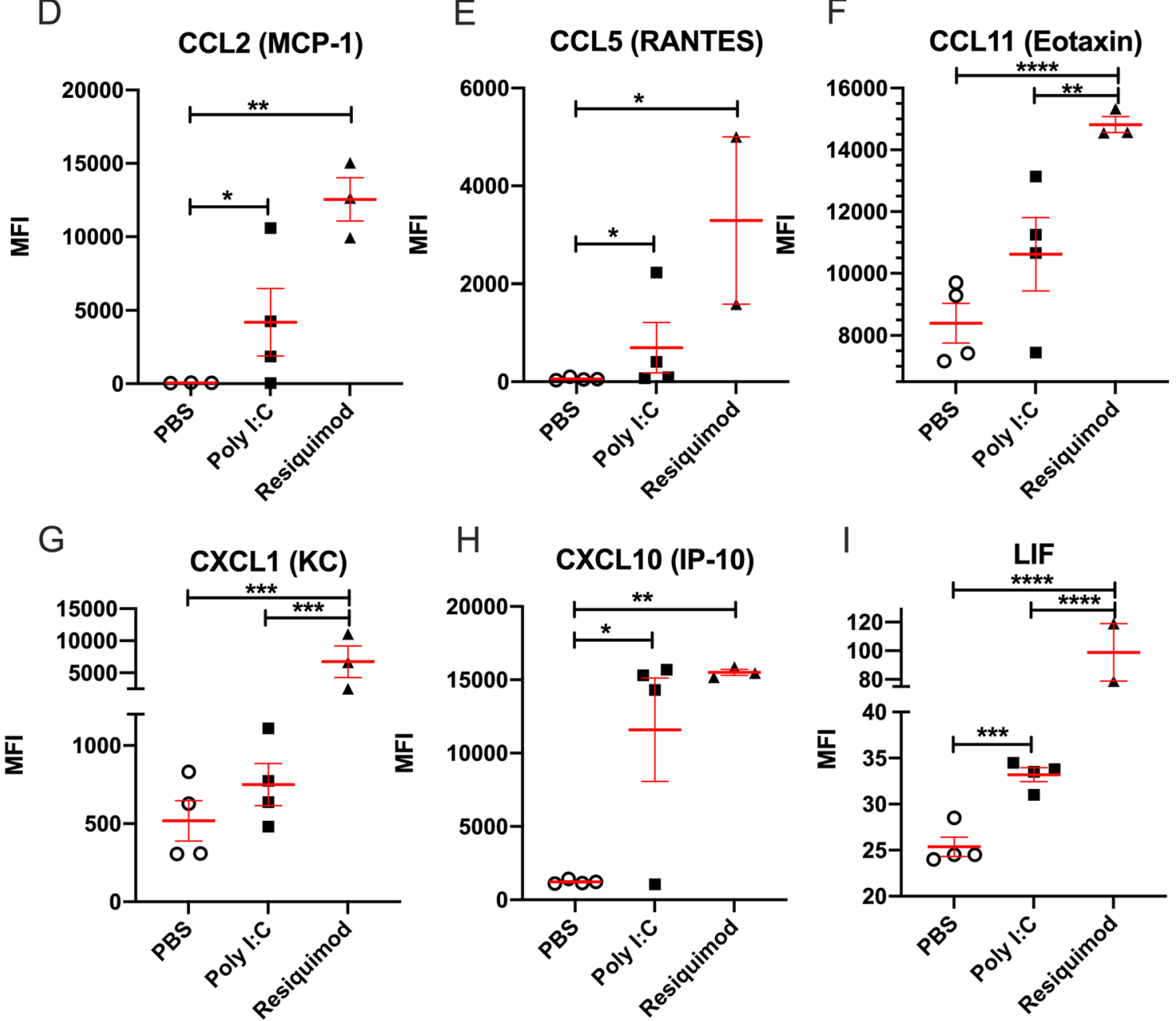

Figure 1. MIA by both poly I:C and resiquimod induces an immune response in maternal plasma. All measured immune molecules were upregulated in the plasma of mothers following resiquimod compared to control (PBS). Poly I:C caused significant upregulation most of measured immune molecules except CCL11 (Eotaxin) and CXCL1 (KC) compared to PBS. The individual dots are shown along with mean \pm SEM. The data were box-cox transformed and analysed by one way-ANOVA, Fisher LSD post-hoc test $(\mathrm{n}=4$ independent samples in each condition; $\left.{ }^{*} p \leq 0.05,{ }^{* *} p \leq 0.005,{ }^{* * *} p \leq 0.001,{ }^{* * *} p \leq 0.0001\right)$. Details of ANOVA F values and $p$ values are provided in Supplementary Table 1. 
A

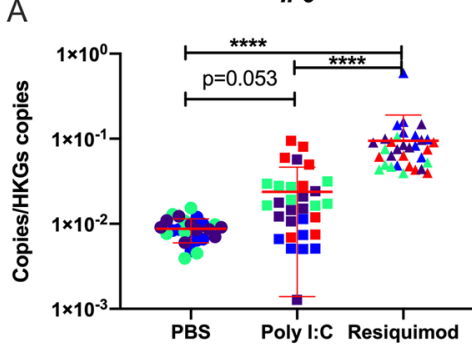

D

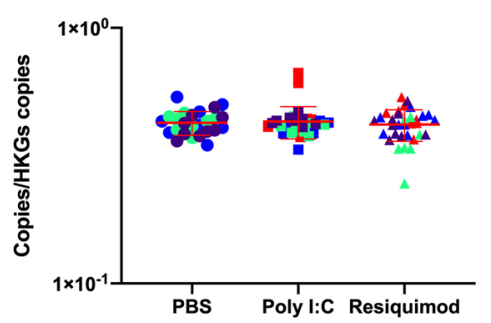

G

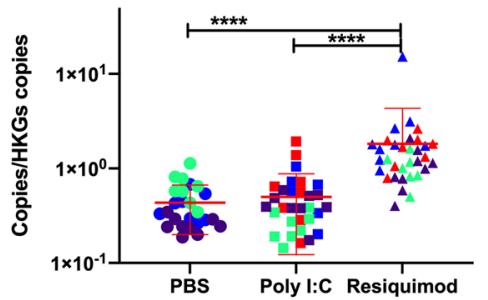

B

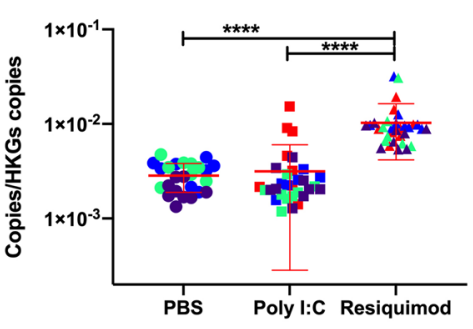

E
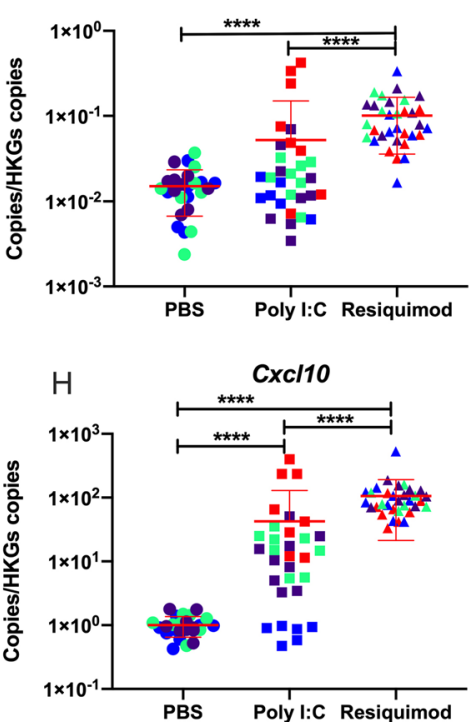

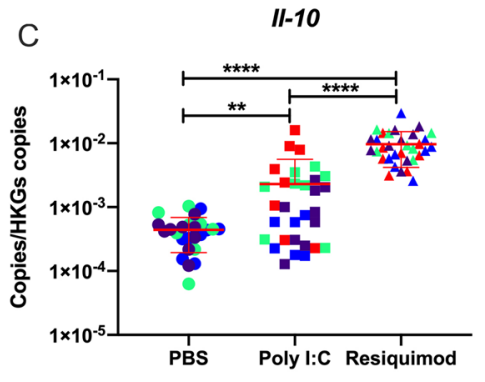

$\mathrm{F}$

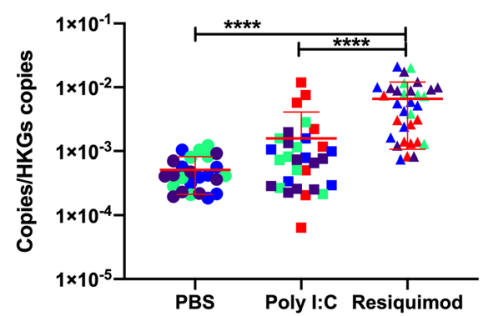

I

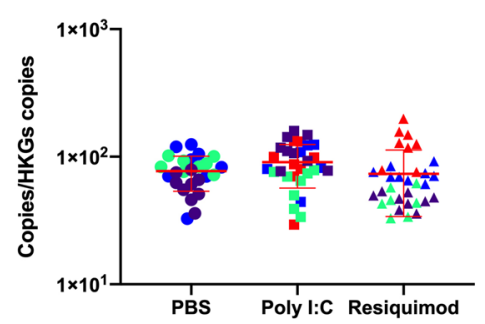

Figure 2. Effect of MIA by poly I:C or resiquimod on immune response in placentae. Placental tissues were collected after $4 \mathrm{~h}$ MIA (PBS, poly I:C (20 mg/kg, LMW), resiquimod (2 mg/kg)). (A,C,H) Il-6, Il-10, and Cxcl10 mRNAs were significantly upregulated by poly I:C and resiquimod compared to PBS. (B,E,F,G) Tnf- $a, C c l 5$, Ccl11, and Cxcl1 were induced by resiquimod, but not by poly I:C compared to PBS. (D,I) CC2 and Cxcl12 were not changed by MIA. Absolute quantification was performed via RT-qPCR and the data were normalised to Gapdh and Tbp. The individual dots are shown along with mean \pm SEM. Colour indicates dams within a single treatment (same colour means "same dam"). The data were log transformed and analysed by two way-ANOVA, Tukey post-hoc test ( $\mathrm{n}=26-33$ independent samples; $\left.{ }^{*} p \leq 0.05,{ }^{* *} p \leq 0.005,{ }^{* * *} p \leq 0.001,{ }^{* * * *} p \leq 0.0001\right)$. Details of ANOVA F values and $p$ values are provided in Supplementary Table 1.

Effects in maternal plasma. Maternal plasma showed evidence of a clear immune response to both poly I:C and resiquimod, with induction of cytokines, including Tnfa and Il-6, and chemokines, including Ccl2 and Ccl5. Maternally-derived Il-6 acting on the placenta, but conceivably originating either in maternal plasma monocytes or in the placenta itself, has been viewed as a key component of MIA effects on the foetus ${ }^{42,43}$. Hence the induction of maternal Il-6 observed here is potentially important for some of the downstream events we detect in the foetal brain. However, our data suggested that maternal plasma IL-6 levels were not correlated with mRNA level changes in placenta and embryo brain samples (data not shown). This suggests that the gene induction in placenta and embryo brain samples is less likely to be caused by maternal plasma IL-6.

At the doses used, however, resiquimod tended to produce larger effects than poly I:C. The variability of the maternal immune response to poly I:C in mice and rats has been highlighted recently ${ }^{14,35,37,44}$, raising concerns with which our data are in agreement. For example, two recent studies of maternal immune activation in rats both report maternal plasma IL-6 increases varying from 0 to $15,000 \%, 3 \mathrm{~h}$ after poly I:C administration ${ }^{14,37}$. In our study, one dam also showed minimal plasma responses to poly I:C for some cytokines and chemokines, despite showing clear placental induction of IL-6 and IL-10. The reasons for this great variability in response, even when using a single batch/source of poly I:C, are not clear. A possibility is that the level of basal TLR3 expression in immune cells is especially sensitive to the history of prior exposure to immune stimuli ${ }^{45-47}$, something which is not necessarily controlled in MIA studies. However, this level of variability is undesirable in an experimental context. It is worth noting that variability (including within litter variability) in the effects of gestational poly I:C on offspring gene expression and behaviour have been highlighted as particularly problematic in the context of preclinical studies ${ }^{44}$. The variation in immune responses that we observed with resiquimod was less marked.

The elevated plasma levels of LIF after exposure to either poly I:C or resiquimod are notable, since maternal LIF is linked to the trans-placental control of foetal neurogenesis ${ }^{48,49}$, and to the modulation of foetal responses 
A
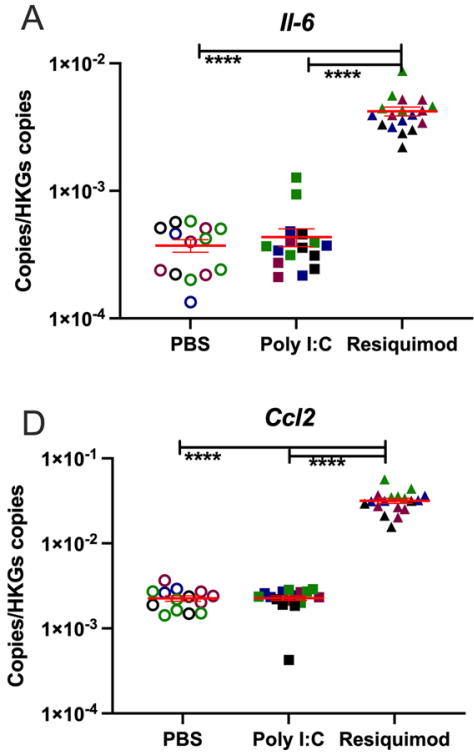

G

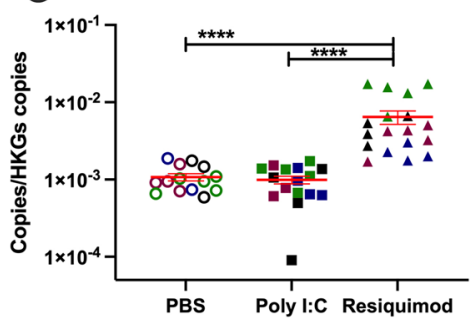

$\mathrm{B}$
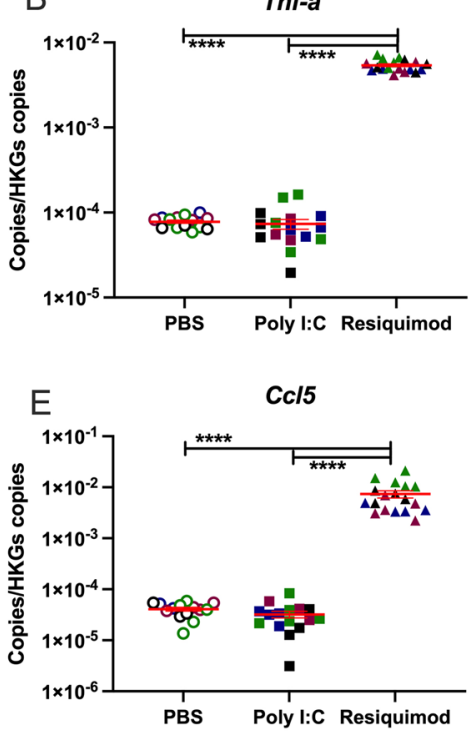

$\mathrm{H}$

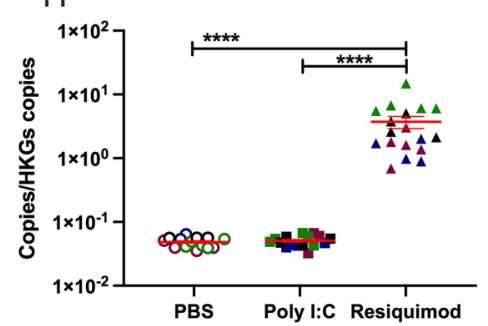

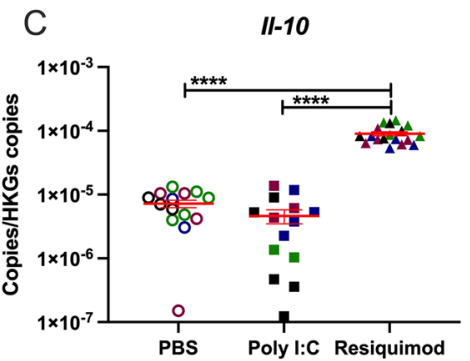
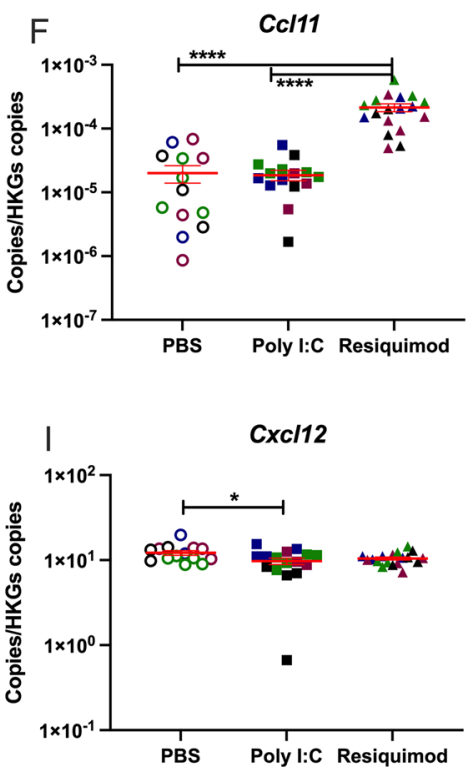

Figure 3. Effect of MIA by poly I:C or resiquimod on immune response in foetal brain. Foetal brain tissues were collected after $4 \mathrm{~h}$ MIA (PBS, poly I:C (20 mg/kg, LMW), resiquimod ( $2 \mathrm{mg} / \mathrm{kg})$ ). (A-H) Cytokine and chemokine mRNAs were significantly induced by resiquimod but not poly I:C compared to control (PBS). (I) Cxcl12 mRNA showed slight changes by MIA. Absolute quantification was performed via RT-qPCR and the data were normalised to Gapdh and Tbp. The individual dots are shown along with mean \pm SEM. Colour indicates dams within a single treatment (same colour means "same dam"). The data were log transformed and analysed by two way-ANOVA, Tukey post-hoc test ( $\mathrm{n}=14-18$ independent samples; ${ }^{*} p \leq 0.05,{ }^{* *} p \leq 0.005,{ }^{* * *} p \leq 0.001$, $\left.{ }_{* * * *} p \leq 0.0001\right)$. Details of ANOVA F values and $p$ values are provided in Supplementary Table 1.

to elevated maternal glucocorticoids ${ }^{50}$. Induction of maternal LIF may be important for the effects of MIA on foetal brain development and psychiatric disease risk.

Effects in placenta. TLRs 3, 4, 7 and 8 are all expressed in placenta ${ }^{51}$, including trophoblasts ${ }^{52}$, and may contribute to the development of preeclampsia ${ }^{53}$, (also a risk factor for schizophrenia in offspring) ( $^{54}$. In previous MIA studies, maternal LPS administration elevated amniotic fluid Tnfa and IL-6 protein after $4 \mathrm{~h}^{55}$. Poly I:C administration increased Tnfa, Il- 6 and Il-10 protein in placenta after $3 \mathrm{~h}^{15}$, increased placental Il-6 mRNA after $3 \mathrm{~h}^{42}$, and increased other cytokine mRNAs (e.g. Tnf $\alpha, \mathrm{Il}-10$ ) to a much lesser degree ${ }^{43}$. We observed placental induction of Il-10 but not Tnf $\alpha$ by poly I:C at $4 \mathrm{~h}$ after administration. The slight discrepancy with respect to Tnf $\alpha$ mRNA may simply reflect the different time point. We previously reported elevated placental Cxcl10 protein $6 \mathrm{~h}$ after poly I:C administration ${ }^{56}$, and here we observed elevated Cxcl10 mRNA levels at $4 \mathrm{~h}$ after administration.

The effects of resiquimod in placenta were greater than those observed with poly I:C for $\mathrm{Il}-6, \mathrm{Il}-10$ and $\mathrm{Cxcl} 10$. Induction of other immune mediators was detected after resiquimod administration, where no poly I:C effects were observed (e.g. Ccl5, Ccl11, Cxcl11, Tnfo, mRNAs). The data show that TLR7/8 stimulation can generate a powerful immune response in placental tissue in mice. It is of interest that human placenta at term ${ }^{57}$, and human umbilical cord blood cells also appear more sensitive to resiquimod than to poly I:C, in terms of induction of IL-6, IL-10 and $\mathrm{TNF}^{58}$ suggesting that the same is true in human tissue. The reasons for this differential responsiveness appear to be unclear, but it is encouraging that the response in mice parallels that in human tissue. In addition, abortions can be a problem with the use of poly I: $\mathrm{C}^{14,15}$. There is no evidence, though, that TLR7/8 stimulation is likely to induce abortions ${ }^{59}$.

From a mechanistic perspective, both a pro-inflammatory (IL-6 and TNFa) and an anti-inflammatory (IL-10) response, as well as an anti-viral (CXCL10) response, have been triggered in the placenta. The more extensive response to resiquimod includes a series of chemokines-CXCL1 and CCL2 are particularly important for 

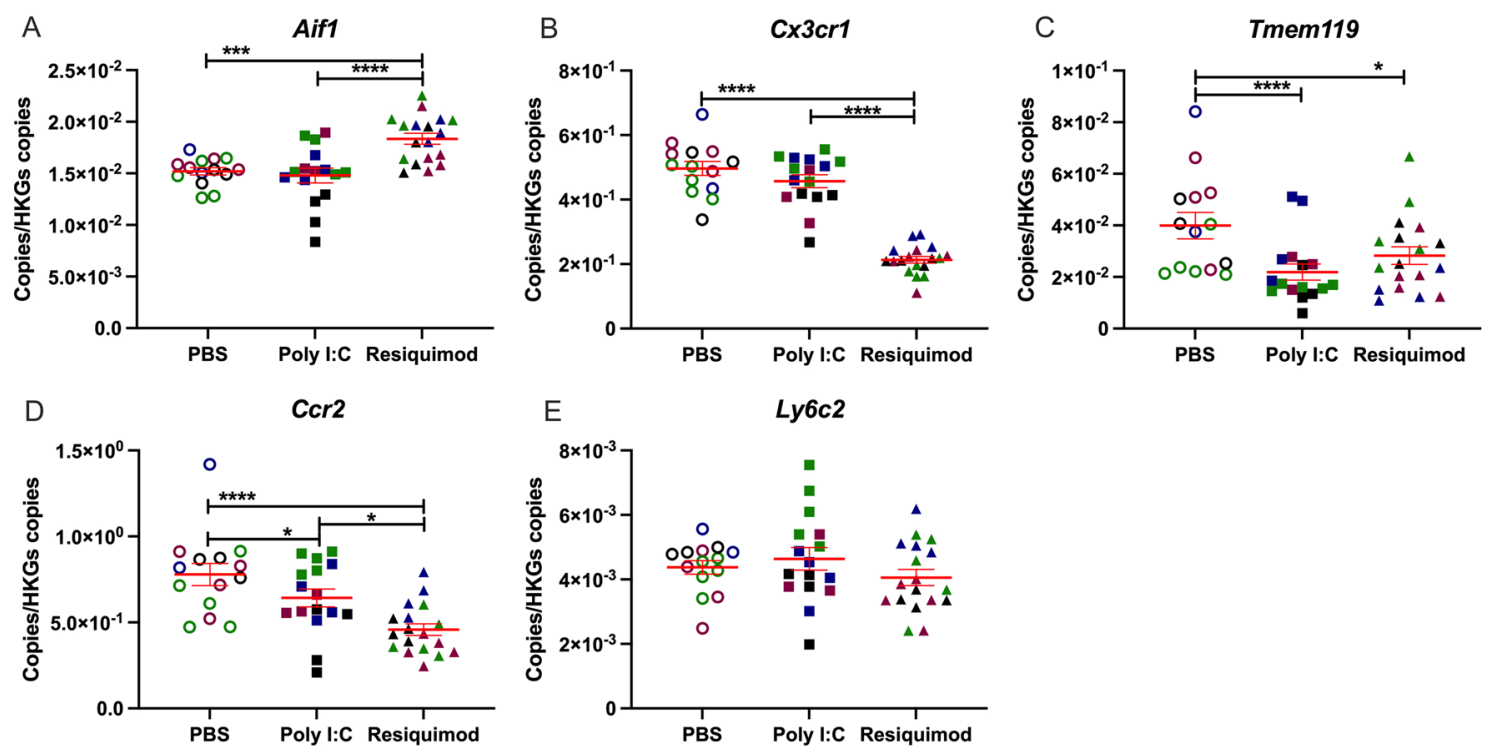

Figure 4. Effect of MIA by poly I:C or resiquimod on microglial markers in foetal brain. Foetal brain tissues were collected after $4 \mathrm{~h}$ MIA (PBS, poly I:C (20 mg/kg, LMW), resiquimod (2 mg/kg)). (A,B) Aif1 was increased by resiquimod, however, $\mathrm{C} x 3 \mathrm{cr} 1$ was downregulated by resiquimod compared to PBS. Both genes were not changed by poly I:C. (C) Tmem 119 was significantly downregulated by MIA compared to PBS. (D, E) Ccr2 was significantly downregulated by MIA compared to PBS, on the other hand, $L y 6 c 2$ was not changed by MIA. Absolute quantification was performed via RT-qPCR and the data were normalised to Gapdh and Tbp. The individual dots are shown along with mean \pm SEM. Colour indicates dams within a single treatment (same colour means "same dam"). The data were log transformed and analysed by two way-ANOVA, Tukey post-hoc test $\left(\mathrm{n}=14-18\right.$ independent samples; $\left.{ }^{*} p \leq 0.05,{ }^{* *} p \leq 0.005,{ }^{* * *} p \leq 0.001,{ }^{* * *} p \leq 0.0001\right)$. Details of ANOVA F values and $p$ values are provided in Supplementary Table 1.

recruiting inflammatory monocytes, and CCL11 is an eosinophil attractant ${ }^{60}$. Hence resiquimod has induced the anticipated anti-viral reaction in the placenta. However, the extent to which these immune response mediators can access the foetal compartment is not clear in most cases (see below).

Effects in foetal brain. In foetal brain, it has been reported that protein levels of IL-1 $\beta$, IL-6, IL-10 and Tnfa were elevated at 3 or $6 \mathrm{~h}$ after maternal poly I:C administration ${ }^{61}$, while another study failed to detect any increase in brain levels of IL- 6 or TNF $\alpha$ h after maternal poly I:C administration ${ }^{62}$. Current concerns about the reproducibility of poly I:C administration have been noted above. Maternal LPS administration, though, elevates foetal brain TNFa and IL-6 protein after $4 \mathrm{~h}$ and $24 \mathrm{~h}^{55}$. In principle, the origin of these cytokines could be from the maternal side of the placenta, but a small increase (<twofold) in Il-6 mRNA has also been detected in foetal brain $3 \mathrm{~h}$ after poly I:C administration, which had normalised by $6 \mathrm{~h}$ after administration ${ }^{42}$. We did not detect any elevations in foetal brain cytokine mRNAs at $4 \mathrm{~h}$ after maternal poly I:C, despite the effects seen in maternal plasma and in placenta. It is possible that small effects of poly I:C on cytokine transcription in foetal brain do occur, but are very transient, and have resolved by $4 \mathrm{~h}$ after drug administration. However, very low relative responses to TLR3 stimulation have also been noted in human foetal tissue ${ }^{58}$. In contrast to the lack of effect of maternal poly I:C on foetal brain cytokines and chemokines, resiquimod produced a large (10-100 fold) stimulation of TNF $, \mathrm{Il}-6, \mathrm{Il}-10, \mathrm{Ccl} 2, \mathrm{Ccl} 5$ and $\mathrm{Cxcl10}$ expression (Fig. 3). Substantial induction of Ccl11 and Cxcl1 mRNAs were also observed. Overall, it is clear from our data that maternal exposure to resiquimod has more substantial effects than poly I:C on the foetal CNS.

Comparing the placental and foetal brain responses, poly I:C up-regulated IL-6 and IL-10 expression in placenta, but not in brain, whereas resiquimod up-regulated these cytokines in both compartments. As noted above, there is evidence that many of the effects of MIA on the foetus (i.e. brain IL- 6 and Cxcl10 induction) are mediated by maternal IL- 6 acting on the placent ${ }^{42,63}$, and there is clear evidence that maternal IL- 6 penetrates into the foetal compartment ${ }^{64,65}$. In this study, the fact that poly I:C increased maternal plasma and placental IL-6 expression, but did not affect all the mediators in the foetal brain affected by resiquimod (including IL-6 and Cxcl10) suggests that other mechanisms are also involved. Therefore, while both agents increased maternal plasma IL-6, our study suggests that not all effects on the foetal brain are mediated via maternal Il-6, and that there are qualitative differences in the foetal brain response to maternal poly I:C and resiquimod administration. One possibility to consider is that resiquimod can penetrate the placental barrier and infiltrate the foetal circulation and brain, while poly I:C cannot. There are no data available concerning the ability of resiquimod to cross the placenta in any species. However, if it can penetrate into the foetal compartment, that would usefully mirror the ability of some of the infectious agents most strongly associated with schizophrenia risk, such as Rubella and Toxoplasma gondii, that do cross the placenta ${ }^{66}$ and affect the foetus directly.

There were some other interesting differences between the foetal brain response and the placental response. For example, $\mathrm{Ccl} 2$ expression was elevated by resiquimod in foetal brain but not in placenta. Clearly this cannot 
reflect different penetration/bioavailability between the two tissue compartments, as resiquimod must pass through the placenta to access the foetal brain. We suggest that the reason is that foetal brain $C c l 2$ expression is from a CNS-specific cell type rather than from immune cells, and specifically from astrocytes, which are present in the developing brain at E12.5 ${ }^{67}$. Indeed, it has been reported that $\mathrm{Ccl} 2$ in the brain is produced basally by astrocytes rather than microglia ${ }^{68,69}$, and that TLR7 stimulation causes Ccl2 release from astrocytes but not from microglia ${ }^{70}$. Thus, the $C c l 2$ mRNA induction may be demonstrating a direct or indirect action of resiquimod on foetal astrocytes.

Evidence for microglial activation in foetal brain. The need to understand the consequences of maternal immune activation (MIA) with ss-virus mimetics becomes particularly important, considering that we have recently demonstrated that the microglial responses to stimulation of TLR3, TLR4 and TLR7 are very different ${ }^{71}$.

While a number of reports suggest microglial activation in foetal brain following MIA in rats or mice ${ }^{35-37}$, there is evidence that maternal poly I:C administration does not evoke activation of foetal brain microglia ${ }^{38}$. We have previously found that LPS, poly I:C and resiquimod produce differing responses in microglial cells in vitro ${ }^{71}$, suggesting that distinct responses to these immune mimetics are also likely to be observed in foetal brain after MIA. TLRs 3, 4, 7 and 8 are all expressed in the CNS at this stage of foetal development ${ }^{72}$. In microglial cells in vitro, sensitivity to TLR3 activation is very low compared to sensitivity to activation of TLR4 or TLR7/8 1 . There is also a differential recruitment of downstream signalling pathways by activated TLR7/8 relative to TLR3 and TLR4. In particular, activation of pro-inflammatory mediator production via the JNK signalling pathway, which is strongly implicated in genetic risk for schizophrenia ${ }^{73}$, is much stronger with TLR7/8 stimulation ${ }^{71}$. Hence there are important differences in the CNS between the consequences of innate immune response to different pathogens.

Resiquimod increased the expression of Aif1 mRNA (encoding the widely used immune cell/ monocyte/ macrophage marker Iba1 $)^{74-76}$, consistent with either activation or proliferation of microglial cells. However, resiquimod decreased the expression of Tmem 119 mRNA and $C x 3 c r 1$ mRNA, in foetal brain. Cx3crl, like Iba1, is commonly used as an immune cell marker, identifying microglia, macrophages and monocytes ${ }^{77-79}$. Tmem119 expression has been recently characterised as being specific to microglia, and not expressed in macrophages or monocytes ${ }^{80}$. However, its biological functions are not fully studied yet.

Microglia respond to immune stimulation with reduced Tmem 119 expression ${ }^{80-83}$ and reduced $C \times 3 \mathrm{cr} 1$ expression $^{83-87}$. In fact, increased expression of Ccl5 and Aif1, and decreased expression of Tmem119 and Cx3cr1, appears to be a highly-characteristic signature of microglial activation ${ }^{88,89}$. Our data therefore are strongly suggestive of microglial activation in the foetal CNS, following maternal TLR7/8, but not TLR3, stimulation. An interesting extension to our study might have been to look for morphological changes indicative of activation in microglia in the foetal tissue, by immunohistochemistry. However, there is consensus that microglia are amoeboid rather than ramified at this stage of foetal development ${ }^{35,90,91}$, and thus morphology is not a useful index of the extent to which they have been activated by an immune stimulus.

The reason for the decreased Ccr2 expression after MIA is less clear. CCR2 is traditionally viewed as a monocyte/macrophage marker, not expressed in microglial cells ${ }^{79,92,93}$. However, the decreased expression of Ccr 2 mRNA after MIA is difficult to reconcile with altered monocyte presence in the foetal brain. There is recent evidence that microglia at E12-14 transiently express low levels of $C c r 2^{94,95}$, which then becomes undetectable later in development. The Ccr2 signal in the foetal brain at E12.5 may therefore derive from microglial cells. TLR4 activation by LPS reportedly decreases CCR2 expression in monocytes ${ }^{83,96-99}$. We favour the interpretation that the diminished $C c r 2$ mRNA expression after poly I:C and resiquimod is a similar phenomenon reflecting immune activation, but in developing microglia. Assuming that there is no penetration of the placenta into the foetal compartment by poly I:C, and maybe resiquimod, the effects on Ccr2 expression are likely to be indirect, mediated by a component of the maternal immune response in blood or placenta.

In contrast to Ccr 2 and $\mathrm{Cx} 3 \mathrm{cr} 1$, Ly6c protein levels are thought to be unchanged by immune stimuli ${ }^{81,82}$. Like Ccr2, Ly6c2 is viewed as a specific monocyte/macrophage marker, not present in microglia, and this indeed appears to be the case whatever the stage of microglial development ${ }^{81,82,89,100,101}$. The lack of change in $L y 6 c 2$ expression that we observe in foetal brain after MIA suggests that there is no overt infiltration of the foetal brain by monocytes.

It is interesting that increased Aif1 mRNA expression, along with decreased expression of $C x 3 \mathrm{cr} 1$ and Tmem 119 mRNAs, has recently been reported in post-mortem temporal cortex from people with schizophrenia ${ }^{102}$. This parallels the changes we observe after maternal resiquimod administration. While this does not necessarily indicate that the altered expression in schizophrenia has been present since early in development, it is at least a possibility that could be considered.

Implications for future research. The use of poly I:C administration to pregnant rats or mice, as a means of modelling the increased risk for schizophrenia caused by in utero exposure to infection, has become widespread $^{13,103,104}$. Recently, however, it has become clear that variability in the characteristics of the poly I:C used are a significant issue ${ }^{14,15}$. We propose that the use of resiquimod as an alternative may offer advantages in terms of reproducibility between laboratories. Furthermore, resiquimod has some conceptual advantages over poly I:C, specifically in relation to modelling schizophrenia risk. Increased risk of schizophrenia is linked with in utero exposure not only to influenza and rubella exposure ${ }^{3,5,105-107}$, (both ss-viruses), but also to bacterial ${ }^{108,109}$ and parasitic (Toxoplasma gondii) infections ${ }^{3,110}$, suggesting that the exact nature of the infectious agent is not a critical factor. However, the epidemiological evidence linking schizophrenia risk to maternal infection specifically by ds-viruses (e.g. CMV, HSV-1) is weak ${ }^{111}$. Conversely, the evidence for maternal ss virus infection as a risk 
factor is strong. Furthermore, Toxoplasma gondii also activates TLR7 $7^{112}$, as do many bacteria ${ }^{113}$. It is also clear that the nature of the immune response in the brain is different to different infectious agents ${ }^{71,114}$.

Hence, until there is a more complete understanding of the mechanisms linking in utero exposure to infection with disease risk, it may be prudent to use a bacterial or ss-virus mimetic rather than poly I:C, for rodent models relating to schizophrenia. Furthermore, since resiquimod, in contrast to poly I:C, is likely to penetrate the placenta and enter the foetal compartment, it may be a much better model for agents such as influenza ${ }^{115}$, rubella ${ }^{7}$ and Toxoplasma gondii ${ }^{66}$, which can also cross the placenta. A direct action of the infectious agent on the foetal brain may be essential for the disease-relevant mechanisms to be triggered. We therefore put resiquimod forward as a useful agent for modelling schizophrenia environmental risk mechanisms in rodents, to reduce experimental variability and maximise construct validity. It will be important for future work to address how gestational exposure to TLR7/8 activation affects the developing foetal brain, and whether there are long-lasting effects on behaviour.

\section{Data availability}

The datasets used and/or analysed during the current study are available from the corresponding author on reasonable request.

Received: 24 September 2021; Accepted: 29 November 2021

Published online: 13 December 2021

\section{References}

1. Brown, A. S. \& Meyer, U. Maternal immune activation and neuropsychiatric illness: A translational research perspective. Am. J. Psychiatry 175, 1073-1083 (2018).

2. al-Haddad, B. J. S. et al. The fetal origins of mental illness. Am. J. Obstet. Gynecol. 221, 549-562 (2019).

3. Brown, A. S. Epidemiologic studies of exposure to prenatal infection and risk of schizophrenia and autism. Dev. Neurobiol. 72, 1272-1276 (2012).

4. Brown, A. S. Prenatal infection as a risk factor for schizophrenia. Schizophr. Bull. 32, 200-202 (2006).

5. Brown, A. S. et al. Prenatal rubella, premorbid abnormalities, and adult schizophrenia. Biol. Psychiat. 49, 473-486 (2001).

6. Mortensen, P. B. et al. Early infections of Toxoplasma gondii and the later development of schizophrenia. Schizophr. Bull. 33, 741-744 (2007).

7. Leung, A. K., Hon, K. \& Leong, K. Rubella (German measles) revisited. Hong Kong Med J 25, 134-141 (2019).

8. Rasmussen, S. A., Jamieson, D. J. \& Uyeki, T. M. Effects of influenza on pregnant women and infants. Am. J. Obstet. Gynecol. 207, S3-S8 (2012).

9. Ostrander, B. \& Bale, J. F. Chapter 6-Congenital and perinatal infections. In Handbook of Clinical Neurology Vol. 162 (ed. de Vries, L. S.) 133-153 (Elsevier, 2019)

10. Wang, M., Zhang, L. \& Gage, F. H. Microglia, complement and schizophrenia. Nat. Neurosci. 22, 333-334 (2019).

11. Meyer, U., Feldon, J. \& Fatemi, S. H. In-vivo rodent models for the experimental investigation of prenatal immune activation effects in neurodevelopmental brain disorders. Neurosci. Biobehav. Rev. 33, 1061-1079 (2009).

12. Meyer, U. Prenatal poly(I:C) exposure and other developmental immune activation models in rodent systems. Biol. Psychiatry 75, 307-315 (2014).

13. Estes, M. L. \& McAllister, A. K. Maternal immune activation: Implications for neuropsychiatric disorders. Science 353, 772-777 (2016).

14. Kowash, H. M. et al. Poly(I:C) source, molecular weight and endotoxin contamination affect dam and prenatal outcomes, implications for models of maternal immune activation. Brain Behav. Immunity 82, 160-166 (2019).

15. Mueller, F. S. et al. Influence of poly(I:C) variability on thermoregulation, immune responses and pregnancy outcomes in mouse models of maternal immune activation. Brain Behav. Immun. 80, 406-418 (2019).

16. Hemmi, H. et al. Small anti-viral compounds activate immune cells via the TLR7 MyD88-dependent signaling pathway. Nat. Immunol. 3, 196-200 (2002).

17. Isobe, Y. et al. Synthesis and biological evaluation of novel 9-substituted-8-hydroxyadenine derivatives as potent interferon inducers. J. Med. Chem. 49, 2088-2095 (2006).

18. Andrade, W. A. et al. Combined action of nucleic acid-sensing Toll-like receptors and TLR11/TLR12 heterodimers imparts resistance to Toxoplasma gondii in mice. Cell Host Microbe 13, 42-53 (2013).

19. Yarovinsky, F. Innate immunity to Toxoplasma gondii infection. Nat. Rev. Immunol. 14, 109-121 (2014).

20. Brown, A. S., Schaefer, C. A., Quesenberry, C. P., Shen, L. \& Susser, E. S. No evidence of relation between maternal exposure to herpes simplex virus type 2 and risk of schizophrenia?. Am. J. Psychiatry 163, 2178-2180 (2006).

21. Missig, G. et al. Sex-dependent neurobiological features of prenatal immune activation via TLR7. Mol. Psychiatry 25, 2330-2341 (2020).

22. Thomson, C. A., McColl, A., Cavanagh, J. \& Graham, G. J. Peripheral inflammation is associated with remote global gene expression changes in the brain. J. Neuroinflamm. 11, 73 (2014).

23. McColl, A., Thomson, C. A., Nerurkar, L., Graham, G. J. \& Cavanagh, J. TLR7-mediated skin inflammation remotely triggers chemokine expression and leukocyte accumulation in the brain. J. Neuroinflamm. 13, 102 (2016).

24. Schön, M. P., Schön, M. \& Klotz, K.-N. The small antitumoral immune response modifier imiquimod interacts with adenosine receptor signaling in a TLR7- and TLR8-independent fashion. J. Investig. Dermatol. 126, 1338-1347 (2006).

25. Wolff, F. et al. Imiquimod directly inhibits Hedgehog signalling by stimulating adenosine receptor/protein kinase A-mediated GLI phosphorylation. Oncogene 32, 5574-5581 (2013).

26. Patinote, C. et al. Agonist and antagonist ligands of Toll-like receptors 7 and 8: Ingenious tools for therapeutic purposes. Eur. J. Med. Chem. 193, 112238 (2020).

27. Michaelis, K. A. et al. Persistent Toll-like receptor 7 stimulation induces behavioral and molecular innate immune tolerance. Brain Behav. Immun. 82, 338-353 (2019).

28. Adzavon, Y. M. et al. TLR7 and TLR8 agonist resiquimod (R848) differently regulates MIF expression in cells and organs. Cytokine 97, 156-166 (2017).

29. Su, Y., Zhang, Z., Trautmann, K., Xu, S. \& Schluesener, H. J. TLR and NOD2 ligands induce cell proliferation in the rat intact spinal cord. J. Neuropathol. Exp. Neurol. 64, 991-997 (2005).

30. Baenziger, S. et al. Triggering TLR7 in mice induces immune activation and lymphoid system disruption, resembling HIVmediated pathology. Blood 113, 377-388 (2009).

31. McAllister, C. S. et al. TLR3, TRIF, and Caspase 8 determine double-stranded RNA-induced epithelial cell death and survival in vivo. J. Immunol. 190, 418 (2013). 
32. Breen, E. J., Polaskova, V. \& Khan, A. Bead-based multiplex immuno-assays for cytokines, chemokines, growth factors and other analytes: Median fluorescence intensities versus their derived absolute concentration values for statistical analysis. Cytokine 71, 188-198 (2015).

33. Breen, E. J., Tan, W. \& Khan, A. The statistical value of raw fluorescence signal in Luminex xMAP based multiplex immunoassays. Sci. Rep. 6, 26996 (2016).

34. Richter, K. R., Nasr, A. N. \& Mexas, A. M. Cytokine concentrations measured by multiplex assays in canine peripheral blood samples. Vet. Pathol. 55, 53-67 (2017).

35. Ozaki, K. et al. Maternal immune activation induces sustained changes in fetal microglia motility. Sci. Rep. 10, 21378-21378 (2020).

36. Prins, J. R., Eskandar, S., Eggen, B. J. L. \& Scherjon, S. A. Microglia, the missing link between maternal immunity and fetal neurodevelopment?. J. Reprod. Immunol. 126, 18-22 (2018).

37. Murray, K. N. et al. Evolution of a maternal immune activation (mIA) model in rats: Early developmental effects. Brain Behav. Immun. 75, 48-59 (2019).

38. Smolders, S. et al. Maternal immune activation evoked by polyinosinic:polycytidylic acid does not evoke microglial cell activation in the embryo. Front. Cell. Neurosci. 9, 301 (2015).

39. Gildawie, K. R., Orso, R., Peterzell, S., Thompson, V. \& Brenhouse, H. C. Sex differences in prefrontal cortex microglia morphology: Impact of a two-hit model of adversity throughout development. Neurosci. Lett. 738, 135381 (2020).

40. Thion, M. S. et al. Microbiome influences prenatal and adult microglia in a sex-specific manner. Cell 172, 500-516 (2018).

41. Cheung, T. T., Weston, M. K. \& Wilson, M. J. Selection and evaluation of reference genes for analysis of mouse (Mus musculus) sex-dimorphic brain development. PeerJ 5, e2909 (2017).

42. Wu, W.-L., Hsiao, E. Y., Yan, Z., Mazmanian, S. K. \& Patterson, P. H. The placental interleukin-6 signaling controls fetal brain development and behavior. Brain Behav. Immun. 62, 11-23 (2017).

43. Hsiao, E. Y. \& Patterson, P. H. Activation of the maternal immune system induces endocrine changes in the placenta via IL-6. Brain Behav. Immun. 25, 604-615 (2011).

44. Mueller, F. S. et al. Behavioral, neuroanatomical, and molecular correlates of resilience and susceptibility to maternal immune activation. Mol. Psychiatry 26, 396-410 (2021).

45. Sirén, J., Pirhonen, J., Julkunen, I. \& Matikainen, S. IFN- $\alpha$ regulates TLR-dependent gene expression of IFN- $\alpha$, IFN- $\beta$, IL-28, and IL-29. J. Immunol. 2005, 174 (1932).

46. Ding, X. et al. TLR4 signaling induces TLR3 up-regulation in alveolar macrophages during acute lung injury. Sci. Rep. 7, 34278-34278 (2017).

47. Heinz, S. et al. Species-specific regulation of Toll-like receptor 3 genes in men and mice. J. Biol. Chem. 278, 21502-21509 (2003).

48. Wright, L. S., Prowse, K. R., Wallace, K., Linskens, M. H. K. \& Svendsen, C. N. Human progenitor cells isolated from the developing cortex undergo decreased neurogenesis and eventual senescence following expansion in vitro. Exp. Cell Res. 312, 2107-2120 (2006).

49. Simamura, E. et al. Maternal leukemia inhibitory factor (LIF) promotes fetal neurogenesis via a LIF-ACTH-LIF signaling relay pathway. Endocrinology 151, 1853-1862 (2010).

50. Ware, C. B., Nelson, A. M. \& Liggitt, D. Late gestation modulation of fetal glucocorticoid effects requires the receptor for leukemia inhibitory factor: An observational study. Reprod. Biol. Endocrinol. 1, 43 (2003).

51. Nishimura, M. \& Naito, S. Tissue-specific mRNA expression profiles of human Toll-like receptors and related genes. Biol. Pharm. Bull. 28, 886-892 (2005).

52. Tangerås, L. H. et al. Functional Toll-like receptors in primary first-trimester trophoblasts. J. Reprod. Immunol. 106, 89-99 (2014).

53. Chatterjee, P. et al. Placental Toll-like receptor 3 and Toll-like receptor $7 / 8$ activation contributes to preeclampsia in humans and mice. PLoS ONE 7, e41884 (2012).

54. Dalman, C., Allebeck, P., Cullberg, J., Grunewald, C. \& Köster, M. Obstetric complications and the risk of schizophrenia: A longitudinal study of a national birth cohort. Arch. Gen. Psychiatry 56, 234-240 (1999).

55. Oskvig, D. B., Elkahloun, A. G., Johnson, K. R., Phillips, T. M. \& Herkenham, M. Maternal immune activation by LPS selectively alters specific gene expression profiles of interneuron migration and oxidative stress in the fetus without triggering a fetal immune response. Brain Behav. Immun. 26, 623-634 (2012).

56. Openshaw, R. L. et al. JNK signalling mediates aspects of maternal immune activation: Importance of maternal genotype in relation to schizophrenia risk. J. Neuroinflamm. 16, 18 (2019).

57. Patni, S. et al. Expression and activity of Toll-like receptors 1-9 in the human term placenta and changes associated with labor at term. Biol. Reprod. 80, 243-248 (2009).

58. Stinson, L. F., Payne, M. S. \& Keelan, J. A. Placental and intra-amniotic inflammation are associated with altered fetal immune responses at birth. Placenta 85, 15-23 (2019).

59. Posobiec, L. M. et al. GSK2245035, a TLR7 agonist, does not increase pregnancy loss in cynomolgus monkeys. J. Reprod. Immunol. 143, 103242 (2021).

60. Sokol, C. L. \& Luster, A. D. The chemokine system in innate immunity. Cold Spring Harbor Perspect. Biol. 7, a016303 (2015).

61. Meyer, U. et al. The time of prenatal immune challenge determines the specificity of inflammation-mediated brain and behavioral pathology. J. Neurosci. 26, 4752-4762 (2006).

62. Abazyan, B. et al. Prenatal interaction of mutant DISC1 and immune activation produces adult psychopathology. Biol. Psychiatry 68, 1172-1181 (2010).

63. Hsiao, E. Y. \& Patterson, P. H. Placental regulation of maternal-fetal interactions and brain development. Dev Neurobiol 72, 1317-1326 (2012).

64. Dahlgren, J., Samuelsson, A. M., Jansson, T. \& Holmang, A. Interleukin-6 in the maternal circulation reaches the rat fetus in mid-gestation. Pediatr. Res. 60, 147-151 (2006).

65. Zaretsky, M. V., Alexander, J. M., Byrd, W. \& Bawdon, R. E. Transfer of inflammatory cytokines across the placenta. Obstet. Gynecol. 103, 546-550 (2004).

66. Robbins, J. R., Zeldovich, V. B., Poukchanski, A., Boothroyd, J. C. \& Bakardjiev, A. I. Tissue barriers of the human placenta to infection with Toxoplasma gondii. Infect. Immun. 80, 418 (2012).

67. Fox, I. J. et al. Developmental expression of glial fibrillary acidic protein mRNA in mouse forebrain germinal zones-Implications for stem cell biology. Dev. Brain Res. 153, 121-125 (2004).

68. Hayashi, M., Luo, Y., Laning, J., Strieter, R. M. \& Dorf, M. E. Production and function of monocyte chemoattractant protein-1 and other beta-chemokines in murine glial cells. J. Neuroimmunol. 60, 143-150 (1995).

69. Peterson, K. E. et al. MCP-1 and CCR2 contribute to non-lymphocyte-mediated brain disease induced by Fr98 polytropic retrovirus infection in mice: Role for astrocytes in retroviral neuropathogenesis. J. Virol. 78, 6449 (2004).

70. Butchi, N. B., Du, M. \& Peterson, K. E. Interactions between TLR7 and TLR9 agonists and receptors regulate innate immune responses by astrocytes and microglia. Glia 58, 650-664 (2010).

71. Kwon, J. et al. Differential effects of toll-like receptor activation and differential mediation by map kinases of immune responses in microglial cells. Cell Mol. Neurobiol. https://doi.org/10.1007/s10571-10021-01127-x (2021).

72. Kaul, D. et al. Expression of Toll-like receptors in the developing brain. PLoS ONE 7, e37767 (2012). 
73. Morris, B. J. \& Pratt, J. A. Novel treatment strategies for schizophrenia from improved understanding of genetic risk. Clin. Genet. 86, 401-411 (2014).

74. Ribeiro, B. M. M. et al. Evidences for a progressive microglial activation and increase in iNOS expression in rats submitted to a neurodevelopmental model of schizophrenia: Reversal by clozapine. Schizophr. Res. 151, 12-19 (2013).

75. Elmore Monica, R. P. et al. Colony-stimulating factor 1 receptor signaling is necessary for microglia viability, unmasking a microglia progenitor cell in the adult brain. Neuron 82, 380-397 (2014).

76. Bruttger, J. et al. Genetic cell ablation reveals clusters of local self-renewing microglia in the mammalian central nervous system. Immunity 43, 92-106 (2015).

77. Harrison, J. K. et al. Role for neuronally derived fractalkine in mediating interactions between neurons and CX3CR1-expressing microglia. Proc. Natl. Acad. Sci. 95, 10896 (1998).

78. Ginhoux, F. et al. Fate mapping analysis reveals that adult microglia derive from primitive macrophages. Science 330, 841-845 (2010).

79. Mizutani, M. et al. The fractalkine receptor but not CCR2 is present on microglia from embryonic development throughout adulthood. J. Immunol. 188, 29 (2012).

80. Bennett, M. L. et al. New tools for studying microglia in the mouse and human CNS. Proc. Natl. Acad. Sci. 113, E1738-E1746 (2016).

81. Ronning, K. E., Karlen, S. J., Miller, E. B. \& Burns, M. E. Molecular profiling of resident and infiltrating mononuclear phagocytes during rapid adult retinal degeneration using single-cell RNA sequencing. Sci. Rep. 9, 4858 (2019).

82. Jordão, M. J. C. et al. Single-cell profiling identifies myeloid cell subsets with distinct fates during neuroinflammation. Science 363, eaat7554 (2019).

83. Sousa, C. et al. Single-cell transcriptomics reveals distinct inflammation-induced microglia signatures. EMBO Rep. 19, e46171 (2018).

84. Haimon, Z. et al. Re-evaluating microglia expression profiles using RiboTag and cell isolation strategies. Nat. Immunol. 19, 636-644 (2018).

85. Boddeke, E. W. G. M. et al. Functional expression of the fractalkine (CX3C) receptor and its regulation by lipopolysaccharide in rat microglia. Eur. J. Pharmacol. 374, 309-313 (1999).

86. Wynne, A. M., Henry, C. J., Huang, Y., Cleland, A. \& Godbout, J. P. Protracted downregulation of CX3CR1 on microglia of aged mice after lipopolysaccharide challenge. Brain Behav. Immun. 24, 1190-1201 (2010).

87. Yosef, N. et al. The phenotypic and functional properties of mouse yolk-sac-derived embryonic macrophages. Dev. Biol. 442, 138-154 (2018).

88. Zhang, S. Z. et al. NG2 glia regulate brain innate immunity via TGF- $\beta 2 / T G F B R 2$ axis. BMC Med. 17, 204 (2019).

89. Butovsky, O. et al. Identification of a unique TGF-beta dependent molecular and functional signature in microglia. Nat. Neurosci. 17, 131-143 (2014).

90. Perry, V. H., Hume, D. A. \& Gordon, S. Immunohistochemical localization of macrophages and microglia in the adult and developing mouse brain. Neuroscience 15, 313-326 (1985).

91. Cengiz, P. et al. Developmental differences in microglia morphology and gene expression during normal brain development and in response to hypoxia-ischemia. Neurochem. Int. 127, 137-147 (2019).

92. Prinz, M., Priller, J., Sisodia, S. S. \& Ransohoff, R. M. Heterogeneity of CNS myeloid cells and their roles in neurodegeneration. Nat. Neurosci. 14, 1227-1235 (2011).

93. Greter, M., Lelios, I. \& Croxford, A. L. Microglia versus myeloid cell nomenclature during brain inflammation. Front. Immunol. 6, 249 (2015).

94. Chen, H.-R. et al. Fate mapping via CCR2-CreER mice reveals monocyte-to-microglia transition in development and neonatal stroke. Sci. Adv. 6, eabb2119 (2020).

95. Kierdorf, K. et al. Microglia emerge from erythromyeloid precursors via Pu.1- and Irf8-dependent pathways. Nat. Neurosci. 16, 273-280 (2013).

96. Sica, A. et al. Bacterial lipopolysaccharide rapidly inhibits expression of C-C chemokine receptors in human monocytes. J. Exp. Med. 185, 969-974 (1997).

97. Heesen, M., Renckens, R., de Vos, A. F., Kunz, D. \& van der Poll, T. Human endotoxemia induces down-regulation of monocyte CC chemokine receptor 2. Clin. Vaccine Immunol. 13, 156 (2006).

98. Weber, C. et al. Downregulation by tumor necrosis factor-alpha of monocyte CCR2 expression and monocyte chemotactic protein-1-induced transendothelial migration is antagonized by oxidized low-density lipoprotein: A potential mechanism of monocyte retention in atherosclerotic lesions. Atherosclerosis 145, 115-123 (1999).

99. Parker, L. C., Whyte, M. K. B., Vogel, S. N., Dower, S. K. \& Sabroe, I. Toll-like receptor (TLR)2 and TLR4 agonists regulate CCR expression in human monocytic cells. J. Immunol. 172, 4977 (2004).

100. DePaula-Silva, A. B. et al. Differential transcriptional profiles identify microglial- and macrophage-specific gene markers expressed during virus-induced neuroinflammation. J. Neuroinflamm. 16, 152 (2019).

101. Bowman, R. L. et al. Macrophage ontogeny underlies differences in tumor-specific education in brain malignancies. Cell Rep. 17, 2445-2459 (2016).

102. Snijders, G. et al. A loss of mature microglial markers without immune activation in schizophrenia. Glia 69, 1251-1267 (2021).

103. Zuckerman, L., Rehavi, M., Nachman, R. \& Weiner, I. Immune activation during pregnancy in rats leads to a postpubertal emergence of disrupted latent inhibition, dopaminergic hyperfunction, and altered limbic morphology in the offspring: A novel neurodevelopmental model of schizophrenia. Neuropsychopharmacology 28, 1778-1789 (2003).

104. Meyer, U., Feldon, J., Schedlowski, M. \& Yee, B. K. Towards an immuno-precipitated neurodevelopmental animal model of schizophrenia. Neurosci. Biobehav. Rev. 29, 913-947 (2005).

105. Mednick, S. A., Machon, R. A., Huttunen, M. O. \& Bonett, D. Adult schizophrenia following prenatal exposure to an influenza epidemic. Arch. Gen. Psychiatry 45, 189-192 (1988).

106. Canetta, S. E. \& Brown, A. S. Prenatal infection, maternal immune activation, and risk for schizophrenia. Transl. Neurosci. 3, 320-327 (2012).

107. Brown, A. S. The environment and susceptibility to schizophrenia. Prog. Neurobiol. 93, 23-58 (2011).

108. Sorensen, H. J., Mortensen, E. L., Reinisch, J. M. \& Mednick, S. A. Association between prenatal exposure to bacterial infection and risk of schizophrenia. Schizophr. Bull. 35, 631-637 (2009).

109. Lee, Y. H. et al. Maternal bacterial infection during pregnancy and offspring risk of psychotic disorders: Variation by severity of infection and offspring sex. Am. J. Psychiatry 177, 66-75 (2020).

110. Brown, A. S. et al. Maternal exposure to toxoplasmosis and risk of schizophrenia in adult offspring. Am. J. Psychiatry 162, 767-773 (2005)

111. Khandaker, G. M., Zimbron, J., Lewis, G. \& Jones, P. B. Prenatal maternal infection, neurodevelopment and adult schizophrenia: A systematic review of population-based studies. Psychol. Med. 43, 239-257 (2013).

112. Yarovinsky, F. \& Sher, A. Toll-like receptor recognition of Toxoplasma gondii. Int. J. Parasitol. 36, 255-259 (2006)

113. Mancuso, G. et al. Bacterial recognition by TLR7 in the lysosomes of conventional dendritic cells. Nat. Immunol. 10, 587-594 (2009). 
114. Olson, J. K. \& Miller, S. D. Microglia initiate central nervous system innate and adaptive immune responses through multiple TLRs. J. Immunol. 173, 3916-3924 (2004).

115. Raj, R. S., Bonney, E. A. \& Phillippe, M. Influenza, immune system, and pregnancy. Reprod. Sci. 21, 1434-1451 (2014).

\section{Acknowledgements}

Not applicable.

\section{Author contributions}

J.K. designed and performed most of the experiments, with assistance from A.M. and M.M. J.C and B.M. assisted with experimental design and data interpretation. J.C provided many of the research reagents. B.M. conceived the study, and wrote the first draft of the paper. All authors provided input to the final version.

\section{Funding}

The research leading to these results received funding from the Wellcome Trust under Grant reference $104025 / \mathrm{Z} / 14 / \mathrm{Z}$.

\section{Competing interests}

The authors declare no competing interests.

\section{Additional information}

Supplementary Information The online version contains supplementary material available at https://doi.org/ 10.1038/s41598-021-03216-9.

Correspondence and requests for materials should be addressed to B.J.M.

Reprints and permissions information is available at www.nature.com/reprints.

Publisher's note Springer Nature remains neutral with regard to jurisdictional claims in published maps and institutional affiliations.

(c) (i) Open Access This article is licensed under a Creative Commons Attribution 4.0 International License, which permits use, sharing, adaptation, distribution and reproduction in any medium or format, as long as you give appropriate credit to the original author(s) and the source, provide a link to the Creative Commons licence, and indicate if changes were made. The images or other third party material in this article are included in the article's Creative Commons licence, unless indicated otherwise in a credit line to the material. If material is not included in the article's Creative Commons licence and your intended use is not permitted by statutory regulation or exceeds the permitted use, you will need to obtain permission directly from the copyright holder. To view a copy of this licence, visit http://creativecommons.org/licenses/by/4.0/.

(C) The Author(s) 2021 